\title{
Adaptive Resource Allocation Scheme for Micromotion Feature Extraction Based on Track-Before-Detect
}

\author{
Yijun Chen $\mathbb{D}^{1,2}$ Qun Zhang $\mathbb{D}^{2,3}$ Ying Luo $\mathbb{D}^{3,4}$ and Tat Soon Yeo ${ }^{4}$ \\ ${ }^{1}$ College of Information Engineering, Engineering University of CAPF, Xi'an 710086, China \\ ${ }^{2}$ Institute of Information and Navigation, Air Force Engineering University, Xi'an 710077, China \\ ${ }^{3}$ Key Laboratory for Information Science of Electromagnetic Waves (Ministry of Education), Fudan University, \\ Shanghai 200433, China \\ ${ }^{4}$ Department of Electrical and Computer Engineering, National University of Singapore, Singapore 117583
}

Correspondence should be addressed to Qun Zhang; zhangqunnus@gmail.com

Received 3 August 2017; Revised 20 December 2017; Accepted 31 December 2017; Published 7 May 2018

Academic Editor: Fanli Meng

Copyright (C) 2018 Yijun Chen et al. This is an open access article distributed under the Creative Commons Attribution License, which permits unrestricted use, distribution, and reproduction in any medium, provided the original work is properly cited.

\begin{abstract}
The micromotion feature extraction method based on track-before-detect (TBD) can save the radar resource and improve the realtime performance of micromotion feature extraction by implementing target detecting, tracking, and micromotion feature extraction simultaneously. Usually, multitargets will exist in different areas, and the limited radar resources should be allocated for different areas to achieve the maximal performance of radar. For single-beam phased array radar, an adaptive resource allocation optimization model is established according to the processing steps of the micromotion feature extraction method based on TBD, and an adaptive resource allocation strategy is proposed. With the method, the radar efficiency can be significantly improved. The effectiveness of the proposed method is demonstrated by simulations.
\end{abstract}

\section{Introduction}

With the beam agile ability, multifunction phased array radar (MFPAR) can control the transmit beam pointing flexibly by selecting the most appropriate target area for observation. Reasonable and effective resource scheduling algorithms are important for exploiting the high adaptive potential of MFPARs [1].

Recently, the study on radar resource allocation has drawn extensive attention of scholars [2-10]. In [2], an adaptive ISAR imaging-considered task scheduling algorithm is proposed, which can improve the radar efficiency by allocating resource for target detecting, tracking, and imaging simultaneously. A time window-based task scheduling approach for MFPAR is proposed in [3], with a simple but predictive heuristic approach, the latency in the schedules of lateness-sensitive tasks can be reduced and the probability of target losses can be lessened. Based on a maximal pulse interleaving technique, an adaptive resource management method for MFPAR is proposed, which can improve the overall capacity of the radar system [4]. Focused on target detecting and tracking, the comparison of two different radar task scheduling methods $[5,6]$ showed that prioritization is a key component to determining overall performance; thus, a knowledge-based fuzzy logic approach for prioritizing radar tasks was introduced and a resource management method is proposed [7]. With the consideration of both the radial cumulative detection probability and tangential cumulative detection probability, a resource scheduling algorithm for phased array radar in searching mode is proposed in [8], which can minimize the total resource consumption under the guarantee of searching performance. In [9], in terms of multitarget tracking, the radar resource is allocated adaptively according to the tracking accuracy, which can make the actual tracking accuracy approach the expected one. Aimed at the micromotion feature extraction of the target, a radar resource allocation method is proposed in [10], where the micromotion feature of the target can be extracted using 
tracking pulses with adaptive update rate; thus, the efficiency of the radar system can be improved.

So far, the existing adaptive resource scheduling methods can be classified into two categories [1-11]: for single-type task and for multitype tasks. For single-type task, the resource allocation optimization models are usually established according to the corresponding performance metrics (such as the detection probability for the detecting tasks and the tracking accuracy for the tracking tasks). For multitype tasks, the source allocation optimization models are usually established according to the priority, the expected execution time, the dwell time, the time window, and data update rate of different type tasks.

To save the radar resource and improve the real-time performance of micromotion feature extraction, we have proposed a novel micromotion feature extraction method based on track-before-detect (TBD) by establishing an information feedback loop [12], with which the micromotion feature parameters of space target can be extracted concurrently with implementing the target detecting and tracking, both the detection probability and micromotion feature extraction precision are much higher than the traditional methods.

However, multitargets may exist in different areas, and the detecting, tracking, and micromotion feature extraction need to be implemented for different areas simultaneously. In this case, the limited radar resources will be not sufficient to be allocated for every area, and then the allocation contradiction of radar resources becomes serious. A reasonable and effective resource allocation strategy is important for exploiting the performance advantages of the micromotion feature extraction method based on TBD [12]. From the available related works, almost all existing resource scheduling methods are aimed at the traditional radar tasks (such as detecting tasks, tracking tasks, and imaging tasks), and for different radar tasks, the resource allocation model is different which depended on the signal processing steps of different radar tasks. Obviously, the signal processing steps of the micromotion feature extraction method based on TBD is different from that of the traditional radar tasks, because it integrates the detecting, tracking, and micromotion feature extraction together. Therefore, the existing adaptive resource scheduling methods will be not usable. In this paper, according to the processing steps of the micromotion feature extraction method in [12], an adaptive resource allocation strategy is proposed, where the performance of detecting, tracking, and micromotion feature extraction is all considered to establish the resource allocation optimization model. By solving the resource allocation optimization model with intelligent optimization algorithms [13, 14]; the reasonable and effective resource allocation can be achieved.

This paper is organized as follows. The brief recap of the micromotion feature extraction method based on TBD is given in Section 2. The relationship between the performances of target detecting, tracking, and micromotion feature extraction and the radar resource consumption is analyzed in Section 3. The resource allocation optimization model is established and solved in Section 4. Simulations are presented in Section 5 and some conclusions are made in the last section.

\section{Micromotion Feature Extraction Method Based on TBD}

In this section, the brief principles of the micromotion feature extraction method based on TBD are reviewed, which is the foundation of this paper. The main idea is that micromotion feature parameters are estimated from the acquired curve information based on TBD technology, and in return, the state transition set of TBD has been updated adaptively according to these extracted feature parameters. A single conical target contains a fixed scatterer $a$ and two slide scatterers $c$ and $d$ have been taken as an example, as shown in Figure 1, where $\vartheta, \omega, \beta, r_{0},|o a|$, $|o b|$, and $v$ are the precession angle, precession frequency, angle between line of sight (LOS) and $z$-axis, radius of the base circle, distance between the target centroid and scatterer $a$, vertical distance between the target centroid and the base circle, and target velocity, respectively.

The projection of $\overrightarrow{\mathbf{o a}}, \overrightarrow{\mathbf{o c}}$, and $\overrightarrow{\mathbf{o d}}$ in the line of sight direction at time $t$ can be represented as $r_{a}(t), r_{c}(t)$, and $r_{d}(t)$, respectively. A feedback loop between micromotion feature extraction and TBD of the target is established, the main steps can be described as follows. The monitoring area is divided into $N_{r} \times N_{\theta}$ grids according to the range and azimuthal angle with stepped increasement of $\Delta r$ and $\Delta \theta$, and each grid is denoted as a state $\mathbf{x}_{k}=\left(i_{k}, j_{k}\right)$. Assume the target consists of $P$ observable scatterers. At the $k$ th scan, for each azimuthal angle $j_{k}$, selecting any $P$ states to form an expanded state $\mathbf{y}_{k}=\left\{\left(i_{k, 1}, j_{k}\right),\left(i_{k, 2}, j_{k}\right), \ldots,\left(i_{k, P}, j_{k}\right)\right\}$, the measured value of $\mathbf{y}_{k}$ is defined as

$$
Z_{k}\left(\mathbf{y}_{k}\right)=\sum_{p=1}^{P} Z_{k}\left(\left(i_{k, p}, j_{k}\right)\right),
$$

where $Z_{k}\left(\left(i_{k, p}, j_{k}\right)\right)$ is the measured value of state $\left(i_{k, p}, j_{k}\right)$. The cumulative energy $I\left(\mathbf{y}_{k}\right)$ can be calculated as

$$
I\left(\mathbf{y}_{k}\right)=Z_{k}\left(\mathbf{y}_{k}\right)+\max _{\mathbf{y}_{k-1} \in \Gamma\left(\mathbf{y}_{k}\right)}\left(I\left(\mathbf{y}_{k-1}\right)\right),
$$

where $\Gamma\left(\mathbf{y}_{k}\right)$ is the state transition set, which contains all the possible state $\mathbf{y}_{k-1}$ which can transit to state $\mathbf{y}_{k}$. Assume that the number of the cumulative energy $I\left(\mathbf{y}_{k}\right)$ which is larger than the detection threshold $T_{\alpha}$ is $Q$. Thus, $Q$ state sequences will be obtained, and each state sequence contains $P$ range trajectories $R_{p}(k)$ and $P$ angle trajectories $\Theta_{p}(k)$. Obviously, for the cone-tip scatterer $a$, the range trajectory should be equal to $r_{a}(t)$, for the cone-base scatterers cand $d$, the estimated range trajectories $R(k)$ should be equal to $r_{c}(t)$ and $r_{d}$ $(t)$, respectively. Therefore, the micromotion feature parameters $\mathbf{P}_{\mathbf{A}}=\left[|o a|, \phi_{0}, \beta, \vartheta, \omega, v,|o b|, r_{0}, R_{c}\right]$ can be extracted via fitting the obtained range trajectory $R_{p}(k)$ according to the mathematical expression of m-D effect $\left(r_{a}(t), r_{c}(t)\right.$, and $\left.r_{d}(t)\right)$ with the Levenberg-Marquardt method. In turn, the extracted micromotion feature parameters are utilized to 


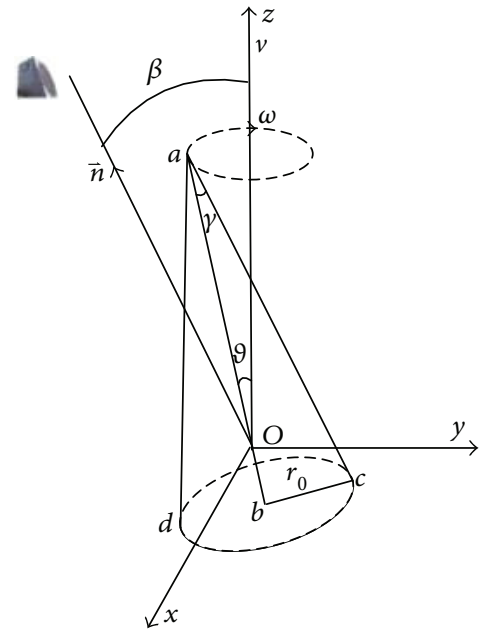

Figure 1: The geometry of conical target with precession.

update the parameters of TBD adaptively. The state $\mathbf{y}_{k}$ will belong to the state transition set $\Gamma\left(\mathbf{y}_{k+1}\right)$ (i.e., $\mathbf{y}_{k} \in \Gamma\left(\mathbf{y}_{k+1}\right)$ ) when it satisfies

$$
\begin{aligned}
& \left|\left(i_{k+1,1}-\frac{N_{r}}{2}\right) \cdot \Delta r+R_{0}-\Delta r_{a}\right|<f_{R}\left(C_{s, \mathbf{y}_{k}}\right) \cdot \Delta r, \\
& \left|j_{k+1}-j_{k}\right|<2 \text {, for scatterer } a, \\
& \left|\left(i_{k+1,2}-\frac{N_{r}}{2}\right) \cdot \Delta r+R_{0}-\Delta r_{c}\right|<f_{R}\left(C_{s, \mathbf{y}_{k}}\right) \cdot \Delta r, \\
& \left|j_{k+1}-j_{k}\right|<2 \text {, for scatterer } c, \\
& \left|\left(i_{k+1,3}-\frac{N_{r}}{2}\right) \cdot \Delta r+R_{0}-\Delta r_{d}\right|<f_{R}\left(C_{s, \mathbf{y}_{k}}\right) \cdot \Delta r, \\
& \left|j_{k+1}-j_{k}\right|<2, \text { for scatterer } d,
\end{aligned}
$$

where $C_{s, \mathbf{y}_{k}}$ represents the consistency of the micromotion feature parameter vectors between the $k-1$ th scan and $k$ th scan, $\Delta r_{a}, \Delta r_{c}$, and $\Delta r_{d}$ are the estimated movement of the three scatters according to the estimated micromotion feature parameters.

In the micromotion feature extraction method based on TBD, the energy accumulation value $I\left(\mathbf{y}_{k}\right)$ and the precision of micromotion feature extraction are both taken into full consideration to declare the presence of a target, where the precision of micromotion feature extraction is described by the consistency of the extracted micromotion feature parameter vectors $C_{s, \mathbf{y}_{k}}$ and fitting error $\widehat{E}_{f, \mathbf{y}_{k}}$. Assume the minimum and maximum total number of scans that are jointly processed in TBD are $K_{N}$ and $K_{M}$, respectively. Two detection thresholds are set: the lower detection threshold $T_{\alpha_{1}}$ and the higher detection threshold $T_{\alpha_{2}}$. In the $k$ th $\left(k \geq K_{N}\right)$ scan, if the cumulative energy $I\left(\mathbf{y}_{k}\right)$ is larger than $T_{\alpha_{2}}$, we declare the presence of a target and the micromotion feature parameters can be obtained via fitting the obtained range trajectories. On the other hand, if the cumulative energy $I\left(\mathbf{y}_{k}\right)$ is larger than $T_{\alpha_{1}}$ and smaller than $T_{\alpha_{2}}$, extract the micromotion feature parameter vector at $k$ th scan, which goes on to cumulate energy for the data of $k+1$ th scan, and extract the micromotion feature parameters at $k+1$ th scan. If $C_{s, \mathbf{y}_{k}}$ and $\widehat{E}_{f, \mathbf{y}_{k}}$ are both relatively small (satisfies $C_{s, \mathbf{y}_{k}}<T_{C}$ and $\widehat{E}_{f, \mathbf{y}_{k}}<T_{E}$ ), we can declare the presence of a target and get the micromotion feature parameters. The energy accumulation is no longer needed. Otherwise, updating the state transition set according to the extracted micromotion feature parameters (3) and the energy accumulation of the data of $k+2$ th scan is needed. Repeat the steps described above until it satisfies $C_{s, \mathbf{y}_{k}}<T_{C}$ and $\widehat{E}_{f, \mathbf{y}_{k}}<T_{E}$ or $I\left(\mathbf{y}_{k}\right)>T_{\alpha_{2}}$, or when it reached the $K_{M}$ th scan.

\section{Performance Analysis with Respect to Resource Consumption}

Usually, multitargets may exist in different areas, and the limited resources should be allocated for each area according to the performance of detecting, tracking, and micromotion feature extraction. That means more resources should be allocated for the area which is of higher probability of target existence, and if the energy accumulation value or the precision of micromotion feature extraction is high enough to declare the presence of the target and obtain the accurate micromotion parameters, less resources will be further allocated to the area. Therefore, to achieve the adaptive resource allocation, the performance of detecting, tracking, and micromotion feature extraction with respect to resource consumption should be analyzed firstly.

The probability density function of cumulative energy $I\left(\mathbf{y}_{k}\right)$ can be calculated, respectively, under two different conditions: one is that only noise exists in the searching gate, the other is that target exists in the searching gate.

The size of $\Gamma\left(\mathbf{y}_{k}\right)$ is denoted as $N_{\Gamma\left(\mathbf{y}_{k}\right)}$ that means there are $N_{\Gamma\left(\mathbf{y}_{k}\right)}$ states in the searching gate which can transit to state $\mathbf{y}_{k}$. If the state $\mathbf{y}_{k}$ is noise, the probability density function of measured value $Z_{k}\left(\mathbf{y}_{k}\right)$ is denoted as $p_{n}(\mathbf{y})$. If the state $\mathbf{y}_{k}$ is the target, the probability density function of measured value $Z_{k}\left(\mathbf{y}_{k}\right)$ is denoted as $p_{t}(\mathbf{y})$.

For the first condition (i.e., only noise exists), all the $N_{\Gamma\left(\mathbf{y}_{k}\right)}$ states in $\Gamma\left(\mathbf{y}_{k}\right)$ are noise states. Assume that $I\left(\mathbf{y}_{k-1}\right)$ is independent and identically distributed for each $\mathbf{y}_{k-1}$, and the distribution function is denoted as $P_{I_{k-1}(\mathrm{y})}$. The distribution function of $\max _{\mathbf{y}_{k-1} \in \Gamma\left(\mathbf{y}_{k}\right)}\left(I\left(\mathbf{y}_{k-1}\right)\right)$ can be represented as $P_{I_{k-1}(\mathbf{y})}^{N_{\Gamma\left(y_{k}\right)}}$, and the corresponding probability density function can be calculated by derivation:

$$
f_{I_{k-1}(\mathbf{y})}=N_{\Gamma\left(\mathbf{y}_{k}\right)} \cdot P_{I_{k-1}(\mathbf{y})}^{N_{\Gamma\left(\mathbf{y}_{k}\right)^{-1}}} \cdot p_{I_{k-1}(\mathbf{y})^{*}}
$$

Without loss of generality, $\max _{\mathbf{y}_{k-1} \in \Gamma\left(\mathbf{y}_{k}\right)}\left(I\left(\mathbf{y}_{k-1}\right)\right)$ and $Z_{k}\left(\mathbf{y}_{k}\right)$ are independent; thus, the probability density function of $I\left(\mathbf{y}_{k}\right)$ can be calculated as

$$
p_{I_{k}(\mathbf{y})}=f_{I_{k-1}(\mathbf{y})} \otimes p_{n}(\mathbf{y})
$$

where $\otimes$ denotes convolution operation. 
For the second condition (i.e., target exists), $\Gamma\left(\mathbf{y}_{k}\right)$ contains $N_{\Gamma\left(\mathbf{y}_{k}\right)}-1$ noise states and one target state. The target state is denoted as $\mathbf{y}_{k-1, T}$, the probability density function of $I\left(\mathbf{y}_{k-1, T}\right)$ is denoted as $\bar{p}_{I_{k-1}(y)}$, and the corresponding distribution function is $\bar{P}_{I_{k-1}(y)}$. The $N_{\Gamma\left(\mathbf{y}_{k}\right)}-1$ noise states are independent and identically distributed with the distribution function of $P_{I_{k-1}(y)}$. Then, the probability density function of $\max _{\mathbf{y}_{k-1} \in \Gamma\left(\mathbf{y}_{k}\right)}\left(I\left(\mathbf{y}_{k-1}\right)\right)$ can be calculated as

$$
\begin{aligned}
\bar{f}_{I_{k-1}(\mathbf{y})}= & \left(N_{\Gamma\left(\mathbf{y}_{k}\right)}-1\right) \cdot P_{I_{k-1}(\mathbf{y})}^{\left.N_{\Gamma\left(\mathbf{y}_{k}\right.}\right)^{-2}} \cdot p_{I_{k-1}(\mathbf{y})} \cdot \bar{P}_{I_{k-1}(\mathbf{y})} \\
& +\bar{p}_{I_{k-1}(\mathbf{y})} \cdot P_{I_{k-1}(\mathbf{y})}^{N_{\Gamma\left(\mathbf{y}_{k}\right)^{-1}}} .
\end{aligned}
$$

Thus, the probability density function of $I\left(\mathbf{y}_{k}\right)$ can be calculated as

$$
p_{I_{k}(\mathbf{y})}=\bar{f}_{I_{k-1}(\mathbf{y})} \otimes p_{t}(\mathbf{y}) \text {. }
$$

In theory, the expression of the probability density function $p_{I_{k}(y)}$ can be obtained according to (4), (5), (6), and (7). However, the analytical expression of $p_{I_{k}(y)}$ is too complicated; thus, it is difficult to be calculated. Therefore, assume $I\left(\mathbf{y}_{k}\right)$ obeys the normal distribution [14], and the mean and variance of $I\left(\mathbf{y}_{k}\right)$ for the two conditions above can be calculated as follows.

For the first condition, assume the measured value $Z_{k}\left(\mathbf{y}_{k}\right)$ of the noise state $\mathbf{y}_{k}$ obeys the standard normal distribution, where the mean $\mu=0$ and the variance $\sigma^{2}=1$. Assume the cumulative energy $I\left(\mathbf{y}_{k-1}\right)$ of the noise state obeys the normal distribution, where the mean and variance are denoted as $\mu_{k-1}$ and $\sigma_{k-1}^{2}$, respectively. The mean and variance of the maximum of $N_{\Gamma\left(\mathbf{y}_{k}\right)}$ random variables which are independent and identically distributed with $N\left(\mu_{k-1}, \sigma_{k-1}^{2}\right)$ are denoted as $\mu_{m 1}$ and $\sigma_{m 1}^{2}$, it holds

$$
\begin{aligned}
\mu_{m 1} & =E\left[\max _{\mathbf{y}_{k-1} \in \Gamma\left(\mathbf{y}_{k}\right)}\left(I\left(\mathbf{y}_{k-1}\right)\right)\right] \\
& =E\left[\sigma_{k-1} \max _{\mathbf{y}_{k-1} \in \Gamma\left(\mathbf{y}_{k}\right)}\left(\frac{I\left(\mathbf{y}_{k-1}\right)-\mu_{k-1}}{\sigma_{k-1}}\right)+\mu_{k-1}\right] \\
& =\sigma_{k-1} E\left[\max _{\mathbf{y}_{k-1} \in \Gamma\left(\mathbf{y}_{k}\right)}\left(\frac{I\left(\mathbf{y}_{k-1}\right)-\mu_{k-1}}{\sigma_{k-1}}\right)\right]+\mu_{k-1} \\
& =\sigma_{k-1} \mu_{\max }\left(N_{\Gamma\left(\mathbf{y}_{k}\right)}\right)+\mu_{k-1},
\end{aligned}
$$

where

$$
\mu_{\max }\left(N_{\Gamma\left(\mathbf{y}_{k}\right)}\right)=\int_{-\infty}^{\infty} N_{\Gamma\left(\mathbf{y}_{k}\right)} x \phi(x) \Phi^{N_{\Gamma\left(y_{k}\right)}}(x) d x,
$$

where $\phi(x)$ and $\Phi(x)$ are the probability density function and the distribution function of the standard normal distribution, respectively. Similarly, it holds

$$
\sigma_{m 1}^{2}=\sigma_{k}^{2} \sigma_{\max }^{2}\left(N_{\Gamma\left(\mathbf{y}_{k}\right)}\right)
$$

where

$$
\begin{aligned}
\mu_{\max }^{2} & \left(N_{\Gamma\left(y_{k}\right)}\right)+\sigma_{\max }^{2}\left(N_{\Gamma\left(y_{k}\right)}\right) \\
& =\int_{-\infty}^{\infty} N_{\Gamma\left(\mathbf{y}_{k}\right)} x^{2} \phi(x) \Phi^{N_{\Gamma\left(y_{k}\right)}-1}(x) d x .
\end{aligned}
$$

According to (2), the recurrence formulas of the mean and variance of $I\left(\mathbf{y}_{k-1}\right)$ can be obtained as

$$
\begin{aligned}
& \mu_{k}=\mu_{m 1}+\mu=\mu_{k-1}+\mu_{\max }\left(N_{\Gamma\left(\mathbf{y}_{k}\right)}\right), \\
& \sigma_{k}^{2}=\sigma_{m 1}^{2}+\sigma^{2}=\sigma_{k-1}^{2} \sigma_{\max }^{2}\left(N_{\Gamma\left(\mathbf{y}_{k}\right)}\right)+1 .
\end{aligned}
$$

At the first scan, $I\left(\mathbf{y}_{k}\right)$ is equal to $Z_{k}\left(\mathbf{y}_{k}\right)$; therefore, it holds $\mu_{0}=0$ and $\sigma_{0}^{2}=1$. Then, the mean and variance of $I\left(\mathbf{y}_{k}\right)$ at each scan can be calculated according to (12) and (13), it means the probability density function of $I\left(\boldsymbol{y}_{k}\right)$ can be obtained and denoted as $f_{H_{0}}\left(I\left(\mathbf{y}_{k}\right)\right) \sim N\left(\mu_{k}, \sigma_{k}^{2}\right)$.

For the second condition, assume the measured value of the target state $\mathbf{y}_{k, T}$ obeys the normal distribution, where the mean is $\bar{\mu}=A$ and the variance is $\bar{\sigma}^{2}=1$. Assume the cumulative energy $I\left(\boldsymbol{y}_{k-1, T}\right)$ of the target state $\mathbf{y}_{k-1, T}$ obeys the normal distribution, where the mean and variance are denoted as $\bar{\mu}_{k-1}$ and $\bar{\sigma}_{k-1}^{2}$, respectively. Assume the cumulative energy $I\left(\boldsymbol{y}_{k-1}\right)$ of the noise state $\mathbf{y}_{k-1}$ obeys the normal distribution, where the mean and variance are denoted as $\mu_{k-1}$ and $\sigma_{k-1}^{2}$, respectively. The defined variables as follows:

$$
\begin{aligned}
\mu_{m 2} & =\sigma_{k-1} \mu_{\max }\left(N_{\Gamma\left(\mathbf{y}_{k}\right)}-1\right)+\mu_{k-1}, \\
\sigma_{m 2}^{2} & =\sigma_{k-1}^{2} \sigma_{\max }^{2}\left(N_{\Gamma\left(y_{k}\right)}-1\right), \\
\Delta & =\mu_{m 2}-\bar{\mu}_{k-1}, \\
\chi & =\sqrt{\sigma_{m 2}^{2}+\bar{\sigma}_{k-1}^{2}}, \\
\phi & =\frac{1}{\sqrt{2 \pi}} \exp \left(-\frac{\Delta^{2}}{2 \chi^{2}}\right), \\
\Phi & =\int_{-\infty}^{\Delta / \chi} \frac{1}{\sqrt{2 \pi}} \exp \left(-\frac{\chi^{2}}{2}\right) \mathrm{dx}, \\
\bar{\Phi} & =1-\Phi .
\end{aligned}
$$

According to (2), the recurrence formulas of the mean and variance of $I\left(\mathbf{y}_{k-1, T}\right)$ can be obtained as

$$
\begin{aligned}
& \bar{\mu}_{k}=\bar{\mu}+\mu_{m 2} \Phi+\bar{\mu}_{k-1} \bar{\Phi}+\chi \phi, \\
& \bar{\sigma}_{k}^{2}=\bar{\sigma}^{2}+\bar{\sigma}_{m 2}^{2} \Phi+\bar{\sigma}_{k-1}^{2} \bar{\Phi}+(\Delta \Phi+\chi \phi)(\Delta \bar{\Phi}-\chi \phi) .
\end{aligned}
$$

At the first scan, it holds $\bar{\mu}_{0}=A$ and $\bar{\sigma}_{0}^{2}=1$. Then, the probability density function of the cumulative energy $I\left(\mathbf{y}_{k, T}\right)$ of target state at each scan can be obtained and denoted as $f_{H 1}\left(I\left(\mathbf{y}_{k}\right)\right) \sim N\left(\bar{\mu}_{k}, \bar{\sigma}_{k}^{2}\right)$.

A binary probability hypothesis is defined as follows:

$$
\begin{aligned}
& H_{0}=\text { target absent } \\
& H_{1}=\text { target present } .
\end{aligned}
$$


Obviously, the prior probability of $I\left(\mathbf{y}_{k}\right)$ can be obtained and denoted as $P\left(I\left(\mathbf{y}_{k}\right) \mid H_{0}\right)$ and $P\left(I\left(\mathbf{y}_{k}\right) \mid H_{1}\right)$ according to $f_{H_{0}}\left(I\left(\mathbf{y}_{k}\right)\right)$ and $f_{H_{1}}\left(I\left(\mathbf{y}_{k}\right)\right)$. When $I\left(\mathbf{y}_{k}\right) \in\left[I\left(\mathbf{y}_{k}\right)-\Delta y / 2\right.$, $\left.I\left(\mathbf{y}_{k}\right)+\Delta y / 2\right]$, where $\Delta y$ is a very small neighborhood radius, $P\left(I\left(\mathbf{y}_{k}\right) \mid H_{0}\right)$ and $P\left(I\left(\mathbf{y}_{k}\right) \mid H_{1}\right)$ can be calculated as

$$
\begin{aligned}
& P\left(I\left(\mathbf{y}_{k}\right) \mid H_{0}\right)=f_{H_{0}}\left(I\left(\mathbf{y}_{k}\right)\right) \cdot \Delta y, \\
& P\left(I\left(\mathbf{y}_{k}\right) \mid H_{1}\right)=f_{H_{1}}\left(I\left(\mathbf{y}_{k}\right)\right) \cdot \Delta y .
\end{aligned}
$$

Further, the posterior probability of $I\left(\mathbf{y}_{k}\right)$ can be calculated according to Bayesian theory

$$
\begin{aligned}
P\left(H_{0} \mid I\left(\mathbf{y}_{k}\right)\right) & =\frac{P\left(I\left(\mathbf{y}_{k}\right) \mid H_{0}\right) P\left(H_{0}\right)}{P\left(I\left(\mathbf{y}_{k}\right) \mid H_{0}\right) P\left(H_{0}\right)+P\left(I\left(\mathbf{y}_{k}\right) \mid H_{1}\right) P\left(H_{1}\right)} \\
& =\frac{f_{H_{0}}\left(I\left(\mathbf{y}_{k}\right)\right) \cdot \Delta y \cdot P\left(H_{0}\right)}{f_{H_{0}}\left(I\left(\mathbf{y}_{k}\right)\right) \cdot \Delta y \cdot P\left(H_{0}\right)+f_{H_{1}}\left(I\left(\mathbf{y}_{k}\right)\right) \cdot \Delta y \cdot P\left(H_{1}\right)},
\end{aligned}
$$

$$
\begin{aligned}
P\left(H_{1} \mid I\left(\mathbf{y}_{k}\right)\right) & =\frac{P\left(I\left(\mathbf{y}_{k}\right) \mid H_{1}\right) P\left(H_{1}\right)}{P\left(I\left(\mathbf{y}_{k}\right) \mid H_{0}\right) P\left(H_{0}\right)+P\left(I\left(\mathbf{y}_{k}\right) \mid H_{1}\right) P\left(H_{1}\right)} \\
& =\frac{f_{H_{1}}\left(I\left(\mathbf{y}_{k}\right)\right) \cdot \Delta y \cdot P\left(H_{1}\right)}{f_{H_{0}}\left(I\left(\mathbf{y}_{k}\right)\right) \cdot \Delta y \cdot P\left(H_{0}\right)+f_{H_{1}}\left(I\left(\mathbf{y}_{k}\right)\right) \cdot \Delta y \cdot P\left(H_{1}\right)},
\end{aligned}
$$

where $P\left(H_{0}\right)$ and $P\left(H_{1}\right)$ are the prior probability of target exist and target not exist, respectively. When $P\left(H_{0}\right)$ and $P\left(H_{1}\right)$ are known, the posterior probability of target existence $P\left(H_{1} \mid I\left(\mathbf{y}_{k}\right)\right)$ can be calculated according to $I\left(\mathbf{y}_{k}\right)$. However, in practice, $P\left(H_{0}\right)$ and $P\left(H_{1}\right)$ are usually unknown. Therefore, the posterior probability of target existence is described by $I\left(\mathbf{y}_{k}\right)$ in this paper. Divide (19) by (20):

$$
\begin{aligned}
F\left(I\left(\mathbf{y}_{k}\right)\right) & \triangleq \frac{P\left(H_{1} \mid I\left(\mathbf{y}_{k}\right)\right)}{P\left(H_{0} \mid I\left(\mathbf{y}_{k}\right)\right)} \\
& =\frac{P\left(I\left(\mathbf{y}_{k}\right) \mid H_{1}\right) P\left(H_{1}\right)}{P\left(I\left(\mathbf{y}_{k}\right) \mid H_{0}\right) P\left(H_{0}\right)} \\
& =\frac{f_{H_{1}}\left(I\left(\mathbf{y}_{k}\right)\right) \cdot \Delta y \cdot P\left(H_{1}\right)}{f_{H_{0}}\left(I\left(\mathbf{y}_{k}\right)\right) \cdot \Delta y \cdot P\left(H_{0}\right)} \\
& =\frac{f_{H_{1}}\left(I\left(\mathbf{y}_{k}\right)\right) \cdot P\left(H_{1}\right)}{f_{H_{0}}\left(I\left(\mathbf{y}_{k}\right)\right) \cdot P\left(H_{0}\right)} .
\end{aligned}
$$

Obviously, the larger the $F\left(I\left(\mathbf{y}_{k}\right)\right)$ is, the higher posterior probability of target existence is. Because of $f_{H_{0}}\left(I\left(\mathbf{y}_{k}\right)\right) \sim$ $N\left(\mu_{k}, \sigma_{k}^{2}\right)$ and $f_{H_{0}}\left(I\left(\mathbf{y}_{k}\right)\right) \sim N\left(\bar{\mu}_{k}, \bar{\sigma}_{k}^{2}\right)$, when $I\left(\mathbf{y}_{k}\right) \in\left[\mu_{k}, \bar{\mu}_{k}\right]$, $f_{H_{0}}\left(I\left(\mathbf{y}_{k}\right)\right)$ decreases monotonically and $f_{H 1}\left(I\left(\mathbf{y}_{k}\right)\right)$ increases monotonically over $I\left(\mathbf{y}_{k}\right)$. Therefore, $F\left(I\left(\mathbf{y}_{k}\right)\right)$ increases monotonically, and we can think that the higher $I\left(\mathbf{y}_{k}\right)$ is, the higher posterior probability of target existence is. When $I\left(\mathbf{y}_{k}\right)<\mu_{k}$ or $I\left(\mathbf{y}_{k}\right)>\bar{\mu}_{k}$, the monotonic of $F\left(I\left(\mathbf{y}_{k}\right)\right)$ is uncertain. Assume that there are $H$ areas that need to be observed, the cumulative energy of the $h$ th area is denoted as $I_{h}\left(\mathbf{y}_{k}\right)$. Normalize $I_{h}\left(\mathbf{y}_{k}\right)$ and define the posterior probability of target existence in each area as

$$
\tilde{P}_{h}\left(\mathbf{y}_{k}\right)= \begin{cases}1, & \max _{h}\left(I_{h}\left(\mathbf{y}_{k}\right)\right)>\bar{\mu}_{k}, I_{h}\left(\mathbf{y}_{k}\right)>\bar{\mu}_{k}, \\ \frac{I_{h}\left(\mathbf{y}_{k}\right)}{\bar{\mu}_{k}}, & \max _{h}\left(I_{h}\left(\mathbf{y}_{k}\right)\right)>\bar{\mu}_{k}, \mu_{k}<I_{h}\left(\mathbf{y}_{k}\right) \leq \bar{\mu}_{k}, \\ \frac{I_{h}\left(\mathbf{y}_{k}\right)}{\max _{h}\left(I_{h}\left(\mathbf{y}_{k}\right)\right)}, & \max _{h}\left(I_{h}\left(\mathbf{y}_{k}\right)\right)<\bar{\mu}_{k}, \mu_{k}<I_{h}\left(\mathbf{y}_{k}\right) \leq \bar{\mu}_{k}, \\ \frac{\mu_{k}}{\min \left(\max _{h}\left(I_{h}\left(\mathbf{y}_{k}\right)\right), \bar{\mu}_{k}\right)}, & I_{h}\left(\mathbf{y}_{k}\right)<\mu_{k} .\end{cases}
$$

In fact, the posterior probability of the target existence in each area is calculated to guide the resource allocation strategy, that is, we want to allocate more resource for the area in which the posterior probability of target existence $\tilde{P}_{h}\left(\mathbf{y}_{k}\right)$ is higher. For the areas in which the cumulative energy satisfies $\mu_{k}<I_{h}\left(\mathbf{y}_{k}\right) \leq \bar{\mu}_{k}$, we can think that the higher $I_{h}\left(\mathbf{y}_{k}\right)$ means the higher posterior probability of target existence due to the monotonic increasing of $F\left(I_{h}\left(\mathbf{y}_{k}\right)\right)$, and more resources should be allocated; thus, $\tilde{P}_{h}\left(\mathbf{y}_{k}\right)$ is in proportion to $I_{h}\left(\mathbf{y}_{k}\right)$ as shown in (22). However, for the areas in which the cumulative energy satisfies $I_{h}\left(\mathbf{y}_{k}\right)<\mu_{k}$, it is hard to determine the posterior probability of target existence according to $I_{h}\left(\mathbf{y}_{k}\right)$ because the monotonic of $F\left(I\left(\mathbf{y}_{k}\right)\right)$ is uncertain. Therefore, for these areas, the equal distribution strategy of radar resource is considered, and the $\tilde{P}_{h}\left(\mathbf{y}_{k}\right)$ of these areas are equal to each other as shown in (22), which means the same resource will be allocated. Similarly, the equal distribution strategy is utilized for the areas in which the cumulative energy satisfies $I_{h}\left(\mathbf{y}_{k}\right)>\bar{\mu}_{k}$.

It should be noticed that the posterior probability of target existence shown as (22) is calculated with the only consideration of the cumulative energy $I_{h}\left(\mathbf{y}_{k}\right)$. However, in the micromotion feature extraction method based on TBD, the energy accumulation value and the precision of micromotion feature extraction are both taken into full consideration to declare the presence of a target. Therefore, the final assessment function of target existence should include two aspects: one is the posterior probability of target existence $\tilde{P}_{h}\left(\mathbf{y}_{k}\right)$ shown in (22) which is calculated according to the energy accumulation value, and the other is the precision of micromotion feature extraction.

The micromotion feature parameters are extracted by fitting the obtained range trajectory $R_{p}(k)$ according to the mathematical expression of $\mathrm{mD}$ effect. Thus, the performance of micromotion feature extraction depends on the target tracking performance. At the $k$ th scan, the range state of target scatters can be represented as $\gamma_{k}=\left\{i_{k, 1}, i_{k, 2}\right.$, $\left.\ldots, i_{k, P}\right\}$, the measured value of $i_{k, p}, p=1,2, \ldots, P$ is denoted as $z_{k, p}, \quad p=1,2, \ldots, P$, and the measured value vector of range state $\gamma_{k}$ can be represented as $z_{k}=\left\{z_{k, 1}\right.$, $\left.z_{k, 2}, \ldots, z_{k, P}\right\}$. All the measured value vectors of the former $k$ scans are denoted as $z_{1: k}=\left\{z_{1}, z_{2}, \ldots, z_{k}\right\}$, then the posterior probability of $\gamma_{k}$ can be represented as

$$
p\left(\gamma_{k} \mid z_{1: k}\right)=\frac{p\left(z_{k} \mid \gamma_{k}\right) \cdot p\left(\gamma_{k} \mid z_{1: k-1}\right)}{p\left(z_{k} \mid z_{1: k-1}\right)}
$$

where 


$$
\begin{aligned}
& p\left(\gamma_{k} \mid z_{1: k-1}\right)=\int p\left(\gamma_{k} \mid \gamma_{k-1}\right) \cdot p\left(\gamma_{k-1} \mid z_{1: k-1}\right) \mathrm{d} \gamma_{k-1}, \\
& p\left(z_{k} \mid z_{1: k-1}\right)=\int p\left(z_{k} \mid \gamma_{k}\right) p\left(\gamma_{k} \mid z_{1: k-1}\right) \mathrm{d} \gamma_{k} .
\end{aligned}
$$

Equations (23), (24), and (25) describe a recursion solving method to obtain $p\left(\gamma_{k} \mid z_{1: k}\right)$, and we can see that the factors which will influence the posterior probability are $p\left(z_{k} \mid \gamma_{k}\right)$ and $p\left(\gamma_{k} \mid \gamma_{k-1}\right)$, where $p\left(z_{k} \mid \gamma_{k}\right)$ is the probability density function of the measured value of range state and $p\left(\gamma_{k} \mid \gamma_{k-1}\right)$ is the probability density function of range state transition. Both $p\left(z_{k} \mid \gamma_{k}\right)$ and $p\left(\gamma_{k} \mid \gamma_{k-1}\right)$ obey normal distribution:

$$
\begin{aligned}
p\left(z_{k} \mid \gamma_{k}\right) & =N\left(z_{k} \mid \gamma_{k}, \sigma_{y}^{2}\right), \\
p\left(\gamma_{k} \mid \gamma_{k-1}\right) & =N\left(\gamma_{k} \mid \gamma_{k-1}+f\left(\mathbf{P}_{\mathbf{A}}\right),(T \sigma)^{2}\right),
\end{aligned}
$$

where

$$
\sigma_{y}^{2}=\left[\begin{array}{cccc}
\sigma_{1,1}^{2} & \rho_{12} \sqrt{\sigma_{1,1}^{2} \cdot \sigma_{2,2}^{2}} & \cdots & \rho_{1 P} \sqrt{\sigma_{1,1}^{2} \cdot \sigma_{P, P}^{2}} \\
\rho_{12} \sqrt{\sigma_{1,1}^{2} \cdot \sigma_{2,2}^{2}} & \sigma_{2,2}^{2} & \cdots & \rho_{2 P} \sqrt{\sigma_{2,2}^{2} \cdot \sigma_{P, P}^{2}} \\
\vdots & \vdots & \vdots & \vdots \\
\rho_{1 P} \sqrt{\sigma_{1,1}^{2} \cdot \sigma_{P, P}^{2}} & \rho_{2 p} \sqrt{\sigma_{2,2}^{2} \cdot \sigma_{P, P}^{2}} & \cdots & \sigma_{P, P}^{2}
\end{array}\right]
$$

$\sigma_{p, p}^{2}$ is the variance of measurement of the $p$ th observable scatterer, and $\rho_{p_{1} p_{2}}$ is the correlation coefficient between the measurements of the $p_{1}$ th and $p_{2}$ th observable scatterer. Without loss of generality, the measurement of each observable scatterer has the same variance; thus, we can denote $\sigma^{2}=\sigma_{p, p}^{2}, \quad p=1,2, \ldots, P$, and the Cramer-Rao lower bound (CRLB) of $\sigma^{2}$ can be represented as

$$
\sigma^{2} \geq\left(\frac{c^{2} / 4}{(1 / 2) \cdot \alpha \cdot \bar{F}^{2}}\right)
$$

where $c$ is the velocity of electromagnetic wave, $\bar{F}^{2}$ is the mean square bandwidth, and $\alpha$ is the SNR of the echo signal, which is proportional to the dwell time $\tau$.

In (27), $T$ is the revisit time interval of observation for the target. $\mathbf{P}_{\mathbf{A}}=\left[|o a|, \phi_{0}, \beta, \vartheta, \omega, v,|o b|, r_{0}, R_{c}\right]$ represents the micromotion feature parameter vector, and $f(\cdot)$ is the state transition function defined as

$$
f\left(\mathbf{P}_{\mathbf{A}}\right)= \begin{cases}F_{A}(t+T)-F_{A}(t), & \text { for scatterer } a \\ F_{C}(t+T)-F_{C}(t), & \text { for scatterer } c \\ F_{D}(t+T)-F_{D}(t), & \text { for scatterer } d\end{cases}
$$

where

$$
\begin{aligned}
F_{A}(t)= & -|o a|\left(\sin \beta \sin \vartheta \sin \left(\omega t+\phi_{0}\right)+\cos \beta \cos \vartheta\right) \\
& -\cos \beta \cdot v \cdot t+R_{c}, \\
F_{C}(t)= & r_{0}-\frac{r_{0}}{2}\left(\cos ^{2} \beta \cos ^{2} \vartheta+\sin ^{2} \beta \sin ^{2} \vartheta \sin ^{2}\left(\omega t+\phi_{0}\right)\right. \\
& \left.+2 \cos \beta \cos \vartheta \sin \beta \sin \vartheta \sin \left(\omega t+\phi_{0}\right)\right) \\
& +|o b|\left(\sin \beta \sin \vartheta \sin \left(\omega t+\phi_{0}\right)+\cos \beta \cos \vartheta\right) \\
& -\cos \beta \cdot v \cdot t+R_{c}, \\
F_{D}(t)=- & r_{0}+\frac{r_{0}}{2}\left(\cos ^{2} \beta \cos ^{2} \vartheta+\sin ^{2} \beta \sin ^{2} \vartheta \sin ^{2}\left(\omega t+\phi_{0}\right)\right. \\
& \left.\quad+2 \cos \beta \cos \vartheta \sin ^{2} \sin \vartheta \sin \left(\omega t+\phi_{0}\right)\right) \\
& +|o b|\left(\sin \beta \sin \vartheta \sin \left(\omega t+\phi_{0}\right)+\cos \beta \cos \vartheta\right) \\
& -\cos \beta \cdot v \cdot t+R_{c} .
\end{aligned}
$$

$(T \sigma)^{2}$ can be represented as

$$
(T \sigma)^{2}=\left[\begin{array}{cccc}
\left(T \sigma_{1,1}^{\prime}\right)^{2} & \rho_{12}^{\prime} \sqrt{\left(T \sigma_{1,1}^{\prime}\right)^{2}\left(T \sigma_{2,2}\right)^{2}} & \cdots & \rho_{1 P}^{\prime} \sqrt{\left(T \sigma_{1,1}^{\prime}\right)^{2}\left(T \sigma_{P, P}^{\prime}\right)^{2}} \\
\rho_{12}^{\prime} \sqrt{\left(T \sigma_{1,1}^{\prime}\right)^{2}\left(T \sigma_{2,2}^{\prime}\right)^{2}} & \left(T \sigma_{2,2}^{\prime}\right)^{2} & \cdots & \rho_{2 P}^{\prime} \sqrt{\left(T \sigma_{2,2}^{\prime}\right)^{2}\left(T \sigma_{P, P}^{\prime}\right)^{2}} \\
\vdots & \vdots & \vdots & \vdots \\
\rho_{1 P}^{\prime} \sqrt{\left(T \sigma_{1,1}^{\prime}\right)^{2}\left(T \sigma_{P, P}^{\prime}\right)^{2}} & \rho_{2 p}^{\prime} \sqrt{\left(T \sigma_{2,2}^{\prime}\right)^{2}\left(T \sigma_{P, P}^{\prime}\right)^{2}} & \cdots & \left(T \sigma_{P, P}^{\prime}\right)^{2}
\end{array}\right],
$$

where $\sigma_{p, p}^{\prime 2}$ is the variance of state noise of the $p$ th observable scatterer with unit time during target moving, and $\rho_{p_{1} p_{2}}^{\prime}$ is the correlation coefficient between the state noises of the $p_{1}$ th and $p_{2}$ th observable scatterer. Similar with $\sigma_{p, p}^{2}$, we can denote $\sigma^{\prime 2}=\sigma_{p, p}^{\prime 2}, \quad p=1,2, \ldots, P$.
On the basis, the tracking error at the $k$ th scan can be calculated as

$$
E_{t, k}=\int p\left(\gamma_{k} \mid z_{1: k}\right) \cdot \gamma_{k} \mathrm{~d} \gamma_{k}-\bar{\gamma}_{k}
$$


where $\bar{\gamma}_{k}$ represents the true range state of the target at the $k$ th scan. Because the micromotion feature parameters are extracted by fitting the obtained range trajectory, the precision of micromotion feature extraction can be described by $E_{t, k}$. The higher $E_{t, k}$ is, the lower precision of micromotion feature extraction is. However, in terms of (33), the true range state of the target at each scan is hard to be known; thus, the precision of micromotion feature extraction can only be described by the consistency of the extracted micromotion feature parameter vectors $C_{s, \mathbf{y}_{k}}$ and fitting error $\widehat{E}_{f, \mathbf{y}_{k}}$, alternatively. The consistency and fitting error of the $h$ th area are denoted as $C_{s, \mathbf{y}_{k}, h}$ and $\widehat{E}_{f, \mathbf{y}_{k}, h}$, respectively. Then, the precision of micromotion feature extraction in each area can be defined as

$$
\tilde{M}_{h}\left(\mathbf{y}_{k}\right)=\frac{1}{2}\left(\tilde{C}_{s, \mathbf{y}_{k}, h}+\tilde{\widetilde{E}}_{f, \mathbf{y}_{k}, h}\right)
$$

where $\tilde{C}_{s, \mathbf{y}_{k}, h}$ and $\widetilde{\widetilde{E}}_{f, \mathbf{y}_{k}, h}$ are the normalized consistency and fitting error.

Based on the analysis above, the assessment function of target existence should be defined with the variables
$\tilde{P}_{h}\left(\mathbf{y}_{k}\right)$ and $\tilde{M}_{h}\left(\mathbf{y}_{k}\right)$, and the limited radar resource should be allocated for the $H$ areas according to the assessment function value of target existence of each area.

In addition, from (12) to (13), (15) to (16), (21) to (22), and (26) to (33), we can get the conclusion that the larger dwell time $\tau$ and smaller revisit time interval $T$ will bring in the better performances of target detection and micromotion feature extraction.

Therefore, in the adaptive resource allocation strategy proposed in this paper, the higher assessment function value of target existence is, the more resource (larger dwell time $\tau$ and smaller revisit time interval $T$ ) will be allocated.

\section{Adaptive Resource Allocation Scheme}

Just as discussed above, the idea of resource allocation is that for the area in which target exists with higher probability, the more resource will be allocated. As a result, the target will be detected with higher probability and the precision of micromotion feature extraction will be higher. According to the performance analysis given in Section 3, the assessment function of target existence can be defined as

$$
J_{h}=\left\{\begin{array}{l}
3, \\
1+\frac{1}{2}\left(\tilde{P}_{h}\left(\mathbf{y}_{k}\right)+\tilde{M}_{h}\left(\mathbf{y}_{k}\right) \cdot f_{m}\left(C_{s, \mathbf{y}_{k}, h}, \widehat{E}_{f, \mathbf{y}_{k}, h}\right)\right), \\
\tilde{P}_{h}\left(\mathbf{y}_{k}\right),
\end{array}\right.
$$

where $J_{h}$ represents the assessment value of target existence in the $h$ th area, and $f_{m}\left(C_{s, \mathbf{y}_{k}, h}, \widehat{E}_{f, \mathbf{y}_{k}, h}\right)$ is a micromotion judgment function defined as

$$
f_{m}\left(C_{s, \mathbf{y}_{k}, h}, \widehat{E}_{f, \mathbf{y}_{k}, h}\right)= \begin{cases}1, & C_{s, \mathbf{y}_{k}, h}>T_{m C}, \widehat{E}_{f, \mathbf{y}_{k}, h}>T_{m E} \\ 0, & \text { else. }\end{cases}
$$

In (35), if the cumulative energy $I_{h}\left(\mathbf{y}_{k}\right)$ is larger than $T_{\alpha_{2}}$ or the micromotion feature extraction results satisfy $C_{s, \mathbf{y}_{k}}<T_{C}$ and $\widehat{E}_{f, \mathbf{y}_{k}}<T_{E}$, we can declare the presence of a target and set the assessment value $J_{h}$ as 3 ; in this case, the radar resources will not be allocated for the area in the next scheduling interval. If the cumulative energy is larger than $T_{\alpha_{1}}$ and smaller than $T_{\alpha_{2}}$, the assessment value $J_{h}$ is determined by the normalized posterior probability of target existence $\tilde{P}_{h}\left(\mathbf{y}_{k}\right)$ and the normalized precision of micromotion feature extraction $\tilde{M}_{h}\left(\mathbf{y}_{k}\right)$. However, a space target can be in two states: micromotion or no micromotion. Thus, the micromotion judgment function $f_{m}\left(C_{s, \mathbf{y}_{k}, h}, \widehat{E}_{f, \mathbf{y}_{k}, h}\right)$ is introduced. If the micromotion feature extraction precision cannot reach the thresholds $T_{m C}$ and $T_{m E}$, only the posterior probability of target existence which is calculated

$$
\begin{aligned}
& \left(I_{h}\left(\mathbf{y}_{k}\right)>T_{\alpha_{2}}\right) \text { or }\left(C_{s, y_{k}, h}<T_{C}, \widehat{E}_{f, \mathbf{y}_{k}, h}<T_{E}\right), \\
& \left(T_{\alpha_{1}}<I_{h}\left(\boldsymbol{y}_{k}\right)<T_{\alpha_{2}}\right),\left(C_{s, \mathbf{y}_{k}, h}>T_{C} \text { or } \widehat{E}_{f, \mathbf{y}_{k}, h}>T_{E}\right), \\
& I_{h}\left(\mathbf{y}_{k}\right)<T_{\alpha_{1}},
\end{aligned}
$$

according to the energy accumulation value will be used to calculate the assessment function value, and only if the micromotion feature extraction precision can reach the thresholds, the micromotion feature extraction results will be considered into the calculation of the assessment function value. In a word, whether the target exhibits or does not exhibits micromotion, the assessment function of the target existence can be calculated adaptively according to the target state. If the cumulative energy $I\left(\mathbf{y}_{k}\right)$ is smaller than $T_{\alpha_{1}}$, the micromotion feature extraction will not be implemented; thus, the assessment function only contains the term of normalized posterior probability of target existence $\tilde{P}_{h}\left(\mathbf{y}_{k}\right)$.

Without loss of generality, we hope that the areas with higher assessment values will be observed with higher probability, the number of areas which can be observed is as more as possible, and the vacant time of radar transmitter is as little as possible. In addition, for the observed areas, the allocated resources for each area are desired to be proportional to the assessment value as far as possible (i.e., the more resources can be allocated for the areas with higher assessment value; thus, the higher performance of target detecting and micromotion feature extraction can be achieved).

Therefore, four objective functions are defined for the resource allocation strategy: 
(1) The sum of assessment value of the areas which can be observed, defined by

$$
\mathrm{SAV}=\sum_{h=1}^{H_{\mathrm{ims}}} J_{h}
$$

where $H_{\mathrm{ims}}$ is the number of areas which can be observed.

(2) The ratio of the areas which can be observed to the total areas which need to be observed, defined by

$$
\mathrm{ROA}=\frac{H_{\mathrm{ims}}}{H},
$$

(3) The resource utilization rate, defined by

$$
\mathrm{RUR}=\frac{\sum_{h}^{H_{\mathrm{ims}}} \sum_{n_{h}=1}^{N_{h}} \tau_{h, n_{h}}}{T_{a}},
$$

where $T_{a}$ is the scheduling time interval, $N_{h}$ is the number of revisits for the $h$ th area, and $\tau_{h, n_{h}}$ is the dwell time of the $n_{h}$ th revisit for the $h$ th area.

(4) The proportion of resource allocation, defined by

$$
\text { PRA }=\operatorname{var}\left(\frac{\sum_{n_{h}=1}^{N_{h}} \tau_{h, n_{h}}}{J_{h}}\right), \quad h=1, \ldots, H_{\mathrm{ims}},
$$

where $\operatorname{var}\left(\sum_{n_{h}=1}^{N_{h}} \tau_{h, n_{h}} / J_{h}, h=1, \ldots, H_{\text {ims }}\right)$ indicates the variance of $\sum_{n_{h}=1}^{N_{h}} \tau_{h, n_{h}} / J_{h}, h=1, \ldots, H_{\text {ims }}$. PRA is used to make the allocated resources for each area to be proportional to the assessment value as far as possible. However, compared with the former three objective functions (i.e., SAV, ROA, and RUR), PRA is less important for the resource allocation strategy.

In this paper, the number of revisits $N_{h}$, the dwell time $\tau_{h, n_{h}}$ of each revisit, and the revisit time interval sequence $T_{h}=\left[T_{1}, T_{2}, \ldots, T_{N_{h}}\right]$ are treated as the optimization variables of the resource allocation. In view of the above objective functions, the stratified resource allocation optimization model with the optimization variables of $N_{h}, \tau_{h, n_{h}}$, and $T_{h}$ is proposed as follows:

$$
\begin{array}{cc}
\max _{\tau_{h, n_{h}}, N_{h}, \boldsymbol{T}_{h}} & \left(L_{1}(\mathrm{SAV}, \mathrm{ROA}, \mathrm{RUR}), L_{2}(-\mathrm{PRA})\right) \\
\text { s.t. } & \sum_{h}^{H_{\mathrm{ims}}} \sum_{n_{h}=1}^{N_{h}} \tau_{h, n_{h}} \leq T_{a}, \\
\forall & \forall \in\left[1,2, \ldots, H_{\mathrm{ims}}\right], J_{h}>1, \\
& f_{m}\left(C_{s, \mathbf{y}_{k}, h}, \widehat{E}_{f, \mathbf{y}_{k}, h}\right)=1, \\
\Delta T_{\max , h}<\frac{1}{4} \cdot \frac{2 \pi}{\omega_{h}},
\end{array}
$$

$$
\begin{gathered}
\forall h \in\left[1,2, \ldots, H_{\mathrm{ims}}\right], \\
J_{h} \leq 1 \text { or } f_{m}\left(C_{s, \mathbf{y}_{k}, h}, \widehat{E}_{f, \mathbf{y}_{k}, h}\right)=0, \\
N_{h}>N_{\mathrm{min}}, \\
\forall h \in\left[1,2, \ldots, H_{\mathrm{ims}}\right], \\
\forall n_{h} \in\left[1,2, \ldots, N_{h}\right], \\
\tau_{h, n_{h}}>\tau_{\min },
\end{gathered}
$$

where $L_{1}$ and $L_{2}$ are the priority marks. Solving $L_{1}$ to obtain the Pareto solution set firstly and then the optimal solution can be obtained by solving $L_{2}$ based on the obtained Pareto solution set of $L_{1}$. Constraint (41a) represents that the total resources allocated for all the observed areas are not beyond the available time resources. In constraint (41b), for the $h$ th area, $\Delta T_{\max , h}$ is the maximum time interval between any two adjacent elements in $T_{h}$, and $\omega_{h}$ is the extracted rotating frequency obtained from the previous scheduling interval. It should be pointed out that if it is the first time to extract the micromotion feature parameter in the scheduling interval, $\omega_{h}$ is set as the maximum possible value of target rotating frequency, which is set as $12 \pi \mathrm{rad} / \mathrm{s}$ in this paper. Generally, fitting the sinusoid-like curve requires that data intervals should be smaller than the quarter of the curve period, which can be guaranteed by constraint (41b). In constraint (41c) and (41d), $\tau_{\min }$ and $N_{\min }$ are the required minimum dwell time and number of revisits for the observed areas.

To get the optimal solution of the resource allocation optimization model shown as (41), the optimization problem of $L_{1}$ should be solved firstly, which is a multiobjective problem (MOP). The nondominated sorting genetic algorithm II (NSGA-II) is one of the most widely applied multiobjective evolutionary algorithms, outperforming other multiobjective evolutionary algorithms such as Pareto-Archived Evolution Strategy (PAES) and Strength-Pareto Evolutionary Algorithm $[15,16]$. Therefore, NSGA-II is adopted to solve $L_{1}$ in this paper.

NSGA-II combines the genetic algorithm, the concept of nondominance, and the crowded distance estimation together $[17,18]$, and the concrete steps of the solving $L_{1}$ based on NSGA-II can be described as follows.

Step 1 (population initialization). In genetic algorithm, each individual of the population presents a problem solution called chromosome [19]. In this paper, the optimization variables are the number of revisits $N_{h}$, the dwell time $\tau_{h, n_{h}}$, and the revisit time interval sequence $T_{h}=\left[T_{1}, T_{2}, \ldots, T_{N_{h}}\right]$ for $H$ areas which need to be observed. The scheduling time interval $T_{a}$ can be divided into $N_{T}=T_{a}$ PRF time segments according to pulse repetition frequency (PRF) of the radar system. Therefore, the chromosome can be described by a time assignment string of length $N_{T}$, and each element of the string includes two parts: the first part represents the area which will be observed at the corresponding time segment, and the second part represents if it is the first pulse of a certain revisit. Obviously, the time assignment string (i.e., chromosome) contains all the information of the number 
TABLE 1: Target parameters.

\begin{tabular}{lccccccccccc}
\hline Parameter & $|o a|(\mathrm{m})$ & $|o b|(\mathrm{m})$ & $r_{0}(\mathrm{~m})$ & $\phi_{0}(\mathrm{rad})$ & $\beta\left(^{\circ}\right)$ & $\vartheta\left(^{\circ}\right)$ & $\omega(\mathrm{rad} / \mathrm{s})$ & $v(\mathrm{~m} / \mathrm{s})$ & $R_{c}(\mathrm{~m})$ & Area number & $A_{h}$ \\
\hline Target 1 & 3.000 & 0.300 & 1.500 & 0.031 & 0.120 & 0.261 & 25.132 & 500 & $4.0 \times 10^{5}$ & 1 & 3.0 \\
Target 2 & 2.800 & 0.200 & 0.900 & 0.142 & 2.535 & 0.124 & 12.566 & 513 & $4.5 \times 10^{5}$ & 2 \\
Target 3 & 2.500 & 0.200 & 0.800 & 0.102 & 0.158 & 0.161 & 37.699 & 493 & $4.2 \times 10^{5}$ & 4 & 3.75 \\
Target 4 & 3.000 & 0.300 & 1.000 & 0.031 & 0.062 & 0.261 & 25.132 & 480 & $4.5 \times 10^{5}$ & 9 & 2.0 \\
Target 5 & 2.800 & 0.200 & 0.900 & 0.142 & 1.827 & 0.124 & 37.699 & 508 & $4.8 \times 10^{5}$ & 6 & 1.25 \\
\hline
\end{tabular}

\begin{tabular}{|c|c|c|c|c|c|c|c|c|c|c|c|c|}
\hline 4 & 2 & 5 & 9 & 1 & 3 & 7 & 8 & 4 & 2 & 1 & 10 & 6 \\
\hline 0 & & 50 & & & & & 150 & & & 200 & & 25 \\
\hline
\end{tabular}

(a)

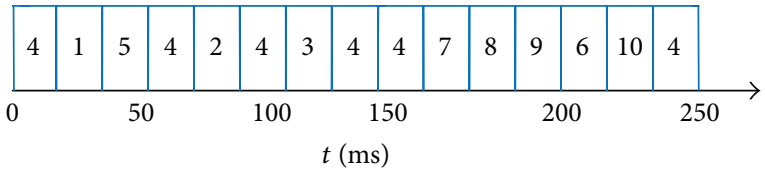

(b)

\begin{tabular}{|c|c|c|c|c|c|c|c|c|c|c|c|c|c|}
\hline 1 & 2 & 1 & 8 & 6 & 1 & 3 & 7 & 1 & 5 & 9 & 1 & 10 & 1 \\
0 & 50 & $t 00$ & $t(\mathrm{~ms})$
\end{tabular}

(c)

\begin{tabular}{|l|l|l|l|l|l|l|l|l|l|l|l|l|l|l|}
\hline 2 & 1 & 3 & 1 & 2 & 8 & 6 & 2 & 1 & 9 & 2 & 10 & 2 & 1 & 2 \\
0 & $100 \quad t(\mathrm{~ms})$
\end{tabular}

(d)

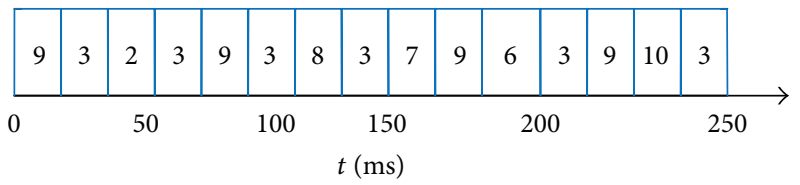

(e)

FIGURE 2: Scheduling timing diagram. (a) Scheduling timing diagram of interval 3. (b) Scheduling timing diagram of interval 7. (c) Scheduling timing diagram of interval 9. (d) Scheduling timing diagram of interval 10. (e) Scheduling timing diagram of interval 15. The meanings of the numbers in the figures represent the area which is observed at the corresponding time segment.

of revisits $N_{h}$, the dwell time $\tau_{h, n_{h}}$ and the revisit time interval sequence $T_{h}=\left[T_{1}, T_{2}, \ldots, T_{N_{h}}\right]$ for $H$ areas. The goal of NSGA-II is to obtain the optimal chromosome which is corresponding to the optimal resource allocation results.

Set the maximum generation number as $L$ and initialize the generation number $l=1$ and the population $G^{(0)}=$ $\left\{g_{1}^{(0)}, g_{2}^{(0)}, \ldots, g_{U}^{(0)}\right\}$ of size $U$.

Step 2 (nondominated sorting and crowding distance calculation). Sorting the population $G^{(l-1)}$ according to the nondomination criteria and assigning the Pareto rank of each

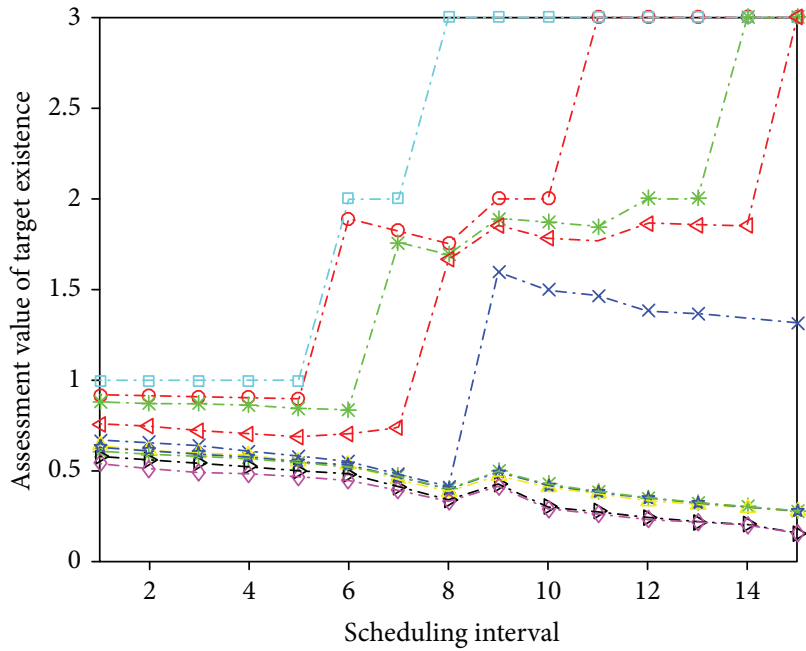

$$
\begin{array}{ll}
-\rightarrow-\text { Area } 1 & -\triangle-\text { Area } 6 \\
-*-\text { Area 2 } & \rightarrow-\text { Area } 7 \\
-*-\text { Area 3 } & -*-\text { Area 8 } \\
-\square-\text { Area } 4 & -\triangleleft-\text { Area } 9 \\
-\rightarrow-\text { Area } 5 & \rightarrow-\text { Area } 10
\end{array}
$$

FIGURE 3: Assessment value of target existence.

individual $g_{u}^{(l-1)}$ as $\operatorname{Rank}\left(g_{u}^{(l-1)}\right)$, which indicates the number of solutions that dominated to the individual [16]. The different nondominated fronts $F_{i}$ can be formed by all the individuals which satisfies $\operatorname{Rank}\left(g_{u}^{(l-1)}\right)=i$. To maintain the population diversity in each nondominated front, the crowding distance of each individual is assigned as $d_{c}\left(g_{u}^{(l-1)}\right)$ [15].

Step 3 (offspring population generation). Generating the offspring population $O^{(l-1)}$ of size $U$ from $G^{(l-1)}$ with the genetic operators. Firstly, the binary tournament selection should be operated, which can be described as two individuals being selected randomly, and then both the Pareto rank and the crowding distance are taken into consideration to judge the winner; the winner is the individual having the smaller Pareto rank; if the Pareto rank is the same for both individuals, the winner is the individual having the higher crowding distance [15]. On this basis, the crossover and mutation operation [20] is taken on the selected individuals, and then the offspring population $O^{(l-1)}$ can be obtained.

Step 4 (recombination and new population generation). Combining $O^{(l-1)}$ and $G^{(l-1)}$ to create a population $\mathrm{NEW}^{(l-1)}$ of size $2 U$, for which the nondominated fronts $F_{i}$ 
TABLE 2: Extracted micromotion feature parameter vector in area 4.

\begin{tabular}{lcccccccccc}
\hline Parameter & $|o a|(\mathrm{m})$ & $|o b|(\mathrm{m})$ & $r_{0}(\mathrm{~m})$ & $\phi_{0}(\mathrm{rad})$ & $\beta\left(^{\circ}\right)$ & $\vartheta\left(^{\circ}\right)$ & $3 \omega(\mathrm{rad} / \mathrm{s})$ & $v(\mathrm{~m} / \mathrm{s})$ & $R_{c}(\mathrm{~m})$ \\
\hline True value & 2.500 & 0.200 & 0.800 & 0.102 & 0.158 & 0.161 & 37.699 & 493.000 & $4.2 \times 10^{5}$ \\
Estimated value & 2.417 & 0.189 & 0.871 & 0.111 & 0.144 & 0.169 & 36.813 & 481.114 & $4.200011 \times 10^{6}$ \\
Error (\%) & 3.32 & 5.50 & 8.87 & 8.82 & 8.86 & 4.97 & 2.35 & 2.41 & 0.00 \\
\hline
\end{tabular}

are formed and the crowding distance of each individual is calculated as described in Step 2. On this basis, the new generation $G^{(l)}$ is filled as follows. Firstly, initialize the generation $G^{(l)}=\varnothing$ and $i=0$, while $\left|G^{(l)}\right|+\left|F_{i}\right|<U$ fills $G^{(l)}=G^{(l)} \cup F_{i}$ and let $i=i+1$. Then, all the individuals in the $F_{i}$ front are sorted descending according to the crowding distance. Finally, the first $U-\left|G^{(h)}\right|-\left|F_{i}\right|$ elements in the sorted $F_{i}$ front are filled into $G^{(l)}$. So far, the new generation $G^{(l)}$ is obtained.

Set $l=l+1$, if $l<L$, repeat Step 2 to Step 4; if $l=L$, iteration ends and the front $F_{0}$ is the Pareto optimal solution set for the optimization problem shown as $L_{1}$ in (41).

On the obtained Pareto optimal solution set for $L_{1}$, the genetic algorithm is adopted to solve the optimization problem shown as $L_{2}$ in (41). As a result, the Pareto optimal solution set of the resource allocation optimization model shown as (41) can be obtained. Finally, the most suitable resource allocation result can be selected from the Pareto optimal solution set by giving weight vector [ $\left.\omega_{1}, \omega_{2}, \omega_{3}\right]$ satisfying $\left(\omega_{1}+\omega_{2}+\omega_{3}=1\right)$ for the three objective functions SAV, ROA, and RUR.

\section{Simulations}

In this section, some simulations are carried out to verify the efficiency of the proposed algorithm. The simulation parameters are set as follows: $K_{N}=6, K_{M}=15, N_{r}=N_{\theta}=$ $1000, \Delta r=0.05 \mathrm{~m}, \Delta \theta=0.005^{\circ}, \phi_{B}=0.15^{\circ}, \varsigma=0.17, T_{C}=$ $0.1, T_{E}=0.1 \mathrm{~m}, \gamma_{1}=1, \gamma_{2}=1.3, P_{n}=10 \mathrm{~dB}, T_{m C}=5, T_{m E}=$ $0.15 \mathrm{~m}, H=10, \mu=0, \sigma^{2}=1, \bar{\mu}_{h}=A_{h}$, and $\bar{\sigma}_{h}^{2}=1$, where $\phi_{B}$ is the beam width, $\varsigma$ is the constant used to control the ratio of intersections number to scans number, $\gamma_{1}$ and $\gamma_{2}$ are the lower detection threshold coefficient and the higher detection threshold coefficient corresponding to $T_{\alpha_{1}}$ and $T_{\alpha_{2}}, P_{n}$ is the noise intensity of the white Gaussian noise, and $A_{h}$ obeys $N(3.3,1)$. Assume 5 areas among the $H=10$ areas are with target existence, and the target parameters are shown in Table 1, where "area number" represents which area the target locates in.

The PRF of the radar system is set as $240 \mathrm{~Hz}$; the scheduling time interval of radar resource is set as $T_{a}=250 \mathrm{~ms}$, and $\tau_{\min }$ and $N_{\min }$ are set as $\tau_{\min }=4 / \mathrm{PRF}$ and $N_{\min }=1$, respectively. The time resources are allocated according to the resource allocation optimization model. By giving weight vector $\omega_{1}=0.4, \omega_{2}=0.4$, and $\omega_{3}=0.2$, the resource allocation results can be acquired, as shown in Figure 2, and the assessment value of target existence in each area $J_{h}$ is shown in Figure 3.

From Figures 2 and 3, we can see that after the 2nd scheduling interval, area 4 is of the highest assessment value of target existence; thus, the most resources are allocated for area 4 in the 3 rd scheduling interval. At the same time, all the areas are observed in the 3rd scheduling interval and allocated resources for each area are almost proportional to the assessment value. After the 6th scheduling interval, both the assessment values of area 4 and area 1 are larger than 1, which means the cumulative energies of these two areas are larger than $T_{\alpha_{1}}$, and the micromotion feature extraction should be implemented in the 7 th scheduling interval. For area 4 and area 1 , it is the first time to extract the micromotion feature parameter; thus, $\omega_{h}$ should be $12 \pi \mathrm{rad} / \mathrm{s}$ in resource allocation optimization model shown as (41). However, the limited radar resources are not enough to be allocated for both area 4 and area 1 to extract the micromotion feature parameter. Because the assessment value of area 4 is larger than that of area 1, more radar resources are allocated for area 4 which satisfies the constraint in (41b), and the micromotion feature extraction can be implemented. For area 1, due to the allocated resources which cannot reach the requirement of sinusoid-like curve fitting, only the energy accumulation can be implemented. After the 8th scheduling interval, the assessment values of area 4 reaches to 3, which means we can declare the presence of a target and get the micromotion feature parameters, and the radar resources will not be allocated for the area in the next scheduling interval. Similarly, in the 9th scheduling interval, the most radar resources are allocated for area 1 which is of the highest assessment value to implement the micromotion feature extraction, and $\omega_{h}$ is also set as $12 \pi$ $\mathrm{rad} / \mathrm{s}$ in the scheduling interval. After the 9th scheduling interval, the estimated $\omega_{h}$ of area 1 is $23.994 \mathrm{rad} / \mathrm{s}$, which is used for the resource allocation in the next scheduling interval and enough resources satisfying the constraint (41b) are allocated for area 1 in the 10th scheduling interval. After the 14th scheduling interval, both the assessment values of area 9 and area 3 are larger than 1; thus, the micromotion feature extraction should be implemented in the next scheduling interval, and enough radar resources are allocated with $\omega_{h}=23.741 \mathrm{rad} / \mathrm{s}$ (obtained in the 14th scheduling interval) for area 9 and $\omega_{h}=12 \pi \mathrm{rad} / \mathrm{s}$ for area 3.

Figure 3 shows that the assessment values of area 4, area 1 , area 2, and area 9 reaching to 3 ; thus, we can declare that the presence of target in area 4, area 1, area 2, and area 9 and the obtained micromotion feature parameters are shown in Tables $2-5$, which are closed to the theoretical value.

To demonstrate the efficiency of the proposed algorithm, the equal distribution method and the traditional resource allocation method [8] are considered for comparison. The performance of target detecting and micromotion feature extraction is compared in Table 6. 
TABLE 3: Extracted micromotion feature parameter vector in area 1.

\begin{tabular}{lcccccccccc}
\hline Parameter & $|o a|(\mathrm{m})$ & $|o b|(\mathrm{m})$ & $r_{0}(\mathrm{~m})$ & $\phi_{0}(\mathrm{rad})$ & $\beta\left(^{\circ}\right)$ & $\vartheta\left(^{\circ}\right)$ & $\omega(\mathrm{rad} / \mathrm{s})$ & $v(\mathrm{~m} / \mathrm{s})$ & $R_{c}(\mathrm{~m})$ \\
\hline True value & 3.000 & 0.300 & 1.500 & 0.031 & 0.120 & 0.261 & 25.132 & 500.000 & $4.0 \times 10^{5}$ \\
Estimated value & 2.846 & 0.324 & 1.592 & 0.028 & 0.111 & 0.251 & 24.173 & 488.651 & $4.000013 \times 10^{5}$ \\
Error (\%) & 5.13 & 8.00 & 6.13 & 9.68 & 7.50 & 3.83 & 3.82 & 2.27 & 0.00 \\
\hline
\end{tabular}

TABLE 4: Extracted micromotion feature parameter vector in area 2.

\begin{tabular}{lccccccccc}
\hline Parameter & $|o a|(\mathrm{m})$ & $|o b|(\mathrm{m})$ & $r_{0}(\mathrm{~m})$ & $\phi_{0}(\mathrm{rad})$ & $\beta\left(^{\circ}\right)$ & $\vartheta\left(^{\circ}\right)$ & $\omega(\mathrm{rad} / \mathrm{s})$ & $v(\mathrm{~m} / \mathrm{s})$ & $R_{c}(\mathrm{~m})$ \\
\hline True value & 2.800 & 0.200 & 0.900 & 0.142 & 2.535 & 0.124 & 12.566 & 513.000 & $4.5 \times 10^{5}$ \\
Estimated value & 2.642 & 0.218 & 0.813 & 0.135 & 2.338 & 0.129 & 13.499 & 499.989 & $4.500019 \times 10^{6}$ \\
Error (\%) & 5.64 & 9.00 & 9.67 & 4.93 & 7.77 & 4.03 & 7.42 & 2.54 & 0.00 \\
\hline
\end{tabular}

TABLE 5: Extracted micromotion feature parameter vector in area 9.

\begin{tabular}{lccccccccc}
\hline Parameter & $|o a|(\mathrm{m})$ & $|o b|(\mathrm{m})$ & $r_{0}(\mathrm{~m})$ & $\phi_{0}(\mathrm{rad})$ & $\beta\left(^{\circ}\right)$ & $\vartheta\left(^{\circ}\right)$ & $\omega(\mathrm{rad} / \mathrm{s})$ & $v(\mathrm{~m} / \mathrm{s})$ & $R_{c}(\mathrm{~m})$ \\
\hline True value & 3.000 & 0.300 & 1.000 & 0.031 & 0.062 & 0.261 & 25.132 & 480.000 & $4.5 \times 10^{5}$ \\
Estimated value & 2.812 & 0.328 & 0.901 & 0.034 & 0.067 & 0.275 & 27.073 & 499.824 & $1.000023 \times 10^{6}$ \\
Error (\%) & 6.27 & 9.33 & 9.90 & 9.68 & 8.06 & 5.36 & 7.72 & 4.13 & 0.00 \\
\hline
\end{tabular}

TABle 6: Performance comparison.

\begin{tabular}{|c|c|c|c|c|c|c|}
\hline & & Target 1 & Target 2 & Target 3 & Target 4 & Target 5 \\
\hline \multirow{2}{*}{ Proposed method } & Detection & Yes & Yes & Yes & Yes & No \\
\hline & Error (\%) & 5.76 & 6.34 & 4.80 & 7.53 & No \\
\hline \multirow{2}{*}{ Equal distribution } & Detection & Yes & No & Yes & No & No \\
\hline & Error (\%) & 236.17 & - & 256.32 & - & - \\
\hline \multirow{2}{*}{ Traditional method } & Detection & No & Yes & Yes & No & No \\
\hline & Error (\%) & - & 218.81 & 223.12 & - & - \\
\hline
\end{tabular}

From Table 6, we can see that 4 targets can be detected with the proposed adaptive resource allocation method, while only 2 targets can be detected with the equal distribution method and traditional resource allocation method. It is because our method is proposed according to the signal processing steps of the micromotion feature extraction method based on TBD, and it utilizes the measurements information and the micromotion feature information together, while the traditional resource allocation method can only utilize the measurement information and the equal distribution method does not utilize any information. What is more, because the constraint in (41b) is not considered in the equal distribution method and traditional resource allocation method, the accurate micromotion feature parameters cannot be obtained; thus, the additional and continuous time resources need to be allocated to implement the micromotion feature extraction. Obviously, our proposed adaptive resource allocation method can detect more targets, obtain higher micromotion feature extraction precision, and improve the radar efficiency.

Finally, the performance of the three resource allocation methods versus the number of observed areas is discussed.
The success of target detection $P_{\mathrm{DS}}$ and the success of micromotion feature extraction $P_{\mathrm{MS}}$ are defined as

$$
\begin{gathered}
P_{\mathrm{DS}}=\frac{K_{\mathrm{DS}}}{K_{a}}, \\
P_{\mathrm{MS}}=\frac{K_{\mathrm{MS}}}{K_{a}},
\end{gathered}
$$

where $K_{a}$ is the number of total targets, $K_{\mathrm{DS}}$ is the number of detected targets, and $K_{\mathrm{MS}}$ is the number of targets for which the estimated error of each micromotion feature parameter is less than $10 \%$. The $P_{\mathrm{DS}}$ and $P_{\mathrm{MS}}$ versus the number of observed areas are shown in Figure 4 , we can see that both $P_{\mathrm{DS}}$ and $P_{\mathrm{MS}}$ of our method are much higher than other methods.

\section{Conclusions}

In this paper, an adaptive resource allocation strategy for micromotion feature extraction method based on trackbefore-detect is proposed. According to the processing steps, a resource allocation optimization model is established based 


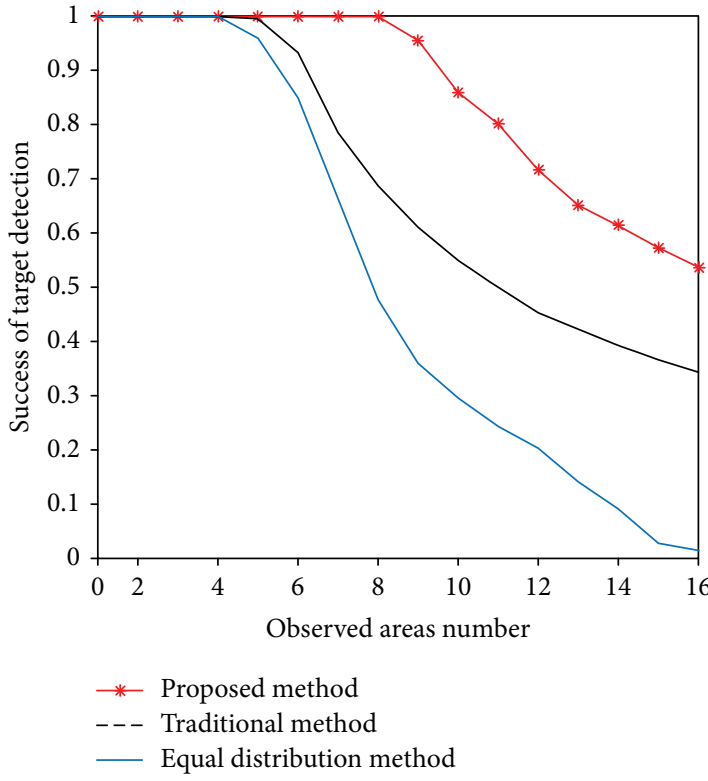

(a) Success of target detection

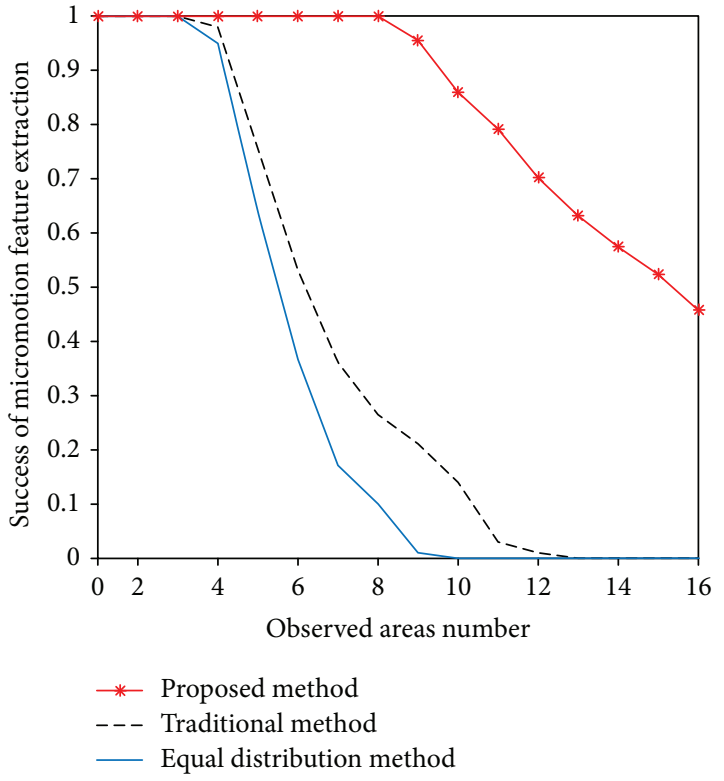

(b) Success of micromotion feature extraction

Figure 4: Performance versus the number of observed areas.

on the analysis of the performances of target detecting, tracking, and micromotion feature extraction with respect to resource consumption. The simulation results show that the proposed resource allocation strategy can improve the radar efficiency significantly.

However, in this paper, the required minimum dwell time and the number of revisits are set as constants according to the traditional detecting tasks and tracking tasks. If they can be adjusted adaptively according to the radar work conditions, the radar efficiency may be further improved. Also, there are many thresholds in the proposed method, which are determined by experiments. If the expression of the performance of target detecting, tracking, and micromotion feature extraction with the thresholds can be derived, the radar efficiency also may be improved. The related contents will be studied in our future work.

\section{Conflicts of Interest}

The authors declare that there are no conflicts of interest regarding the publication of this article.

\section{Acknowledgments}

This work was supported in part by the National Natural Science Foundation of China under Grant nos. 61631019, 61471386, and 61571457 and in part by Ministry of Education, Singapore under Grant no. MOE2016-T2-1-070.

\section{References}

[1] W. Xiangli, Y. Wei, and K. Lingiiang, "Joint beam selection and dwell time allocation for multi-target tracking in phased array radar system," Journal of Radars, vol. 6, no. 6, pp. 602610, 2017.
[2] Y. Chen, Q. Zhang, N. Yuan, Y. Luo, and H. Lou, "An adaptive ISAR-imaging-considered task scheduling algorithm for multi-function phased array radars," IEEE Transactions on Signal Processing, vol. 63, no. 19, pp. 5096-5110, 2015.

[3] D. S. Jang, H. L. Choi, and J. E. Roh, “A time-window-based task scheduling approach for multi-function phased array radars," in 2011 11th International Conference on Control, Automation and Systems, pp. 1250-1255, Gyeonggi-do, Republic of Korea, October 2011.

[4] C. Jie, W. Lei, Z. Wei, and Z. Ke, "Multifunction phased radar resource management via maximal pulse interleaving technique," Arabian Journal for Science and Engineering, vol. 38, no. 11, pp. 3081-3091, 2013.

[5] J. M. Butler, Multi-Function Radar Tracking and Control, [Ph.D. thesis], University College London, London, UK, 1998.

[6] A. J. Orman, C. N. Potts, A. K. Shahani, and A. R. Moore, "Scheduling for a multifunction phased array radar system," European Journal of Operational Research, vol. 90, no. 1, pp. 13-25, 1996.

[7] S. Miranda, C. Baker, K. Woodbridge, and H. Griffiths, "Knowledge-based resource management for multifunction radar: a look at scheduling and task prioritization," IEEE Signal Processing Magazine, vol. 23, no. 1, pp. 66-76, 2006.

[8] J. Wu and T. Cheng, "Research on phased array radar resource management in searching mode," MATEC Web of Conferences, vol. 59, article 07005, 2016.

[9] S. Qing, Z. Qin, and T. Jianfeng, "Research on resource management of phased-array radar in target tracking," in 2015 International Conference on Automation, Mechanical Control and Computational Engineering, pp. 150-155, Jinan, China, January 2015.

[10] Y. Chen, Q. Zhang, C. Ma, Y. Luo, and T. S. Yeo, "Micromotion feature extraction of radar target using tracking pulses with adaptive pulse repetition frequency adjustment," Journal of Applied Remote Sensing, vol. 8, no. 1, article 083569, 2014. 
[11] J. Yan, H. Liu, B. Jiu, B. Chen, Z. Liu, and Z. Bao, "Simultaneous multibeam resource allocation scheme for multiple target tracking," IEEE Transactions on Signal Processing, vol. 63, no. 12, pp. 3110-3122, 2015.

[12] Y. Chen, Q. Zhang, Y. Luo, and T. S. Yeo, "Micromotion feature extraction of space target based on track-before-detect," Journal of Sensors, vol. 2017, Article ID 8723042, 14 pages, 2017.

[13] L. Li, X. Chen, and X. Xiang, "An intelligent optimization algorithm for joint MCS and resource block allocation in LTE femtocell downlink with QoS guarantees," in The 2014 5th International Conference on Game Theory for Networks, pp. 1-6, Beijing, China, November 2014.

[14] Z. X. Cong, P. C. Li, J. X. Ou, and Z. W. Peng, "A new method of power system load forecasting based on intelligent optimization algorithm," Advanced Materials Research, vol. 10701072, pp. 1439-1445, 2014.

[15] S. Bandyopadhyay and R. Bhattacharya, "Solving multiobjective parallel machine scheduling problem by a modified NSGA-II," Applied Mathematical Modelling, vol. 37, no. 10-11, pp. 6718-6729, 2013.

[16] A. Martínez-Vargas, J. Domínguez-Guerrero, and Á. G. Andrade, "Application of NSGA-II algorithm to the spectrum assignment problem in spectrum sharing networks," Applied Soft Computing, vol. 39, pp. 188-198, 2016.

[17] Y. Yusoff, M. S. Ngadiman, and A. M. Zain, "Overview of NSGA-II for optimizing machining process parameters," Procedia Engineering, vol. 15, pp. 3978-3983, 2011.

[18] M. Shaygan, A. Alimohammadi, and A. Mansourian, "Spatial multi-objective optimization approach for land use allocation using NSGA-II," IEEE Journal of Selected Topics in Applied Earth Observations and Remote Sensing, vol. 7, no. 3, pp. 906-916, 2014.

[19] Y. J. Chen, Q. Zhang, Y. Luo, and Y. A. Chen, "Measurement matrix optimization for ISAR sparse imaging based on genetic algorithm," IEEE Geoscience and Remote Sensing Letters, vol. 13, no. 12, pp. 1875-1879, 2016.

[20] P. Vivekanandan, M. Rajalakshmi, and R. Nedunchezhian, "An intelligent genetic algorithm for mining classification rules in large datasets," Computing and Informatics, vol. 32, no. $1,2013$. 


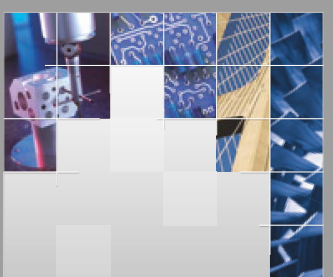

\section{Enfincering}
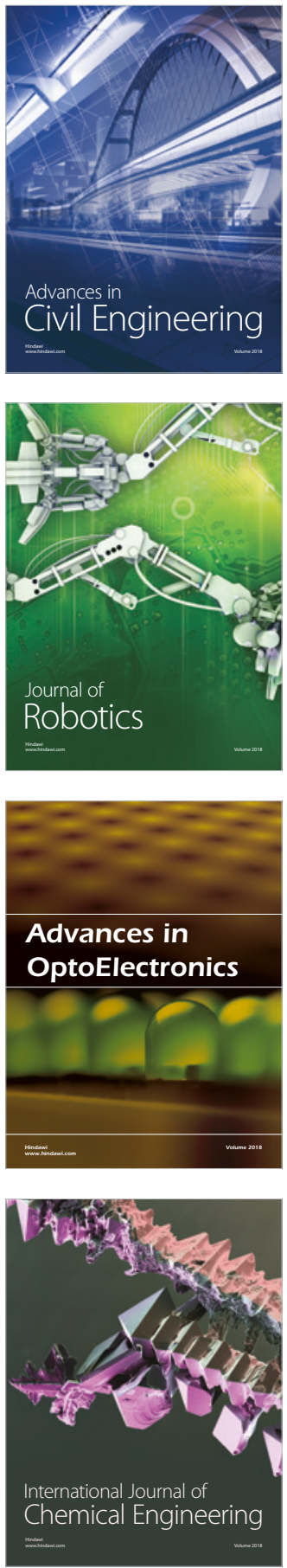

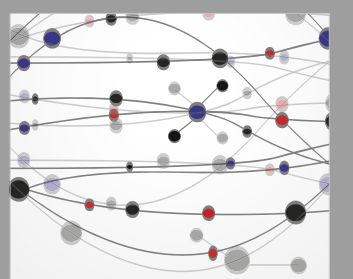

\section{Rotating \\ Machinery}

The Scientific World Journal

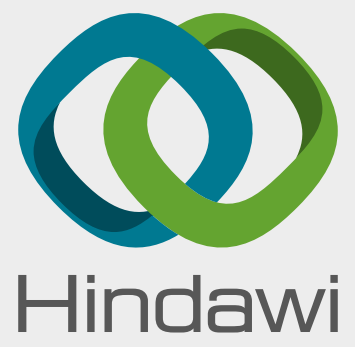

Submit your manuscripts at

www.hindawi.com
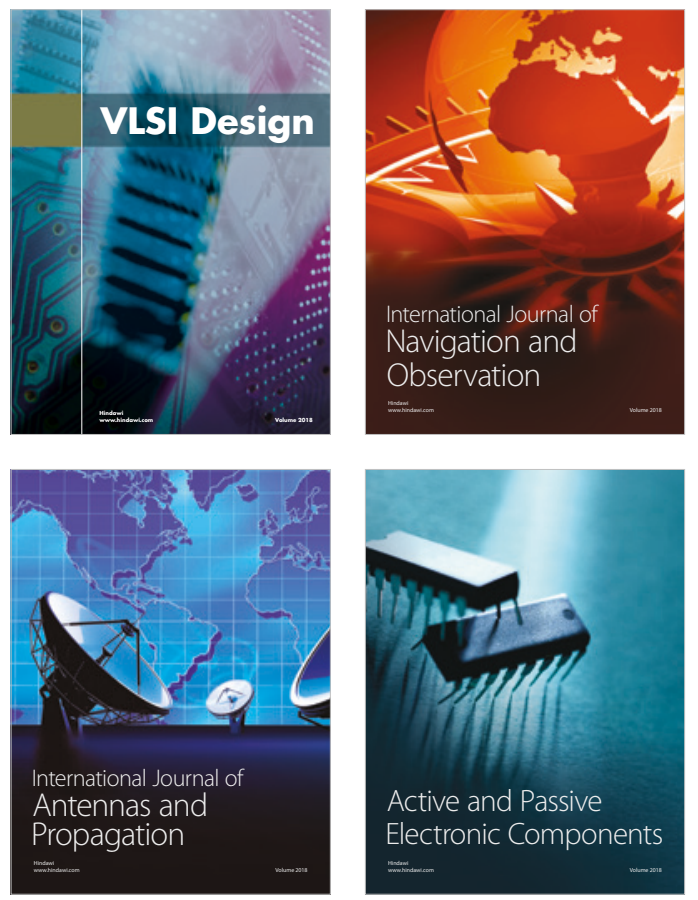
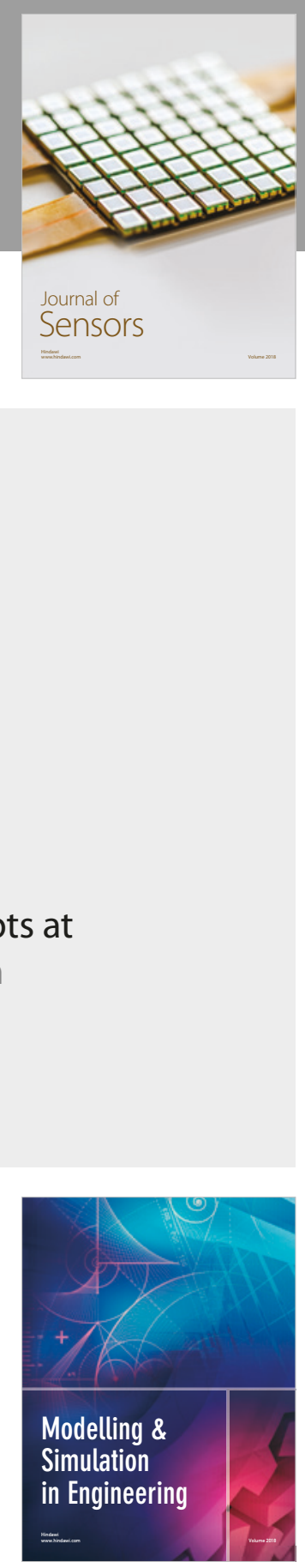

\section{Advances \\ Multimedia}
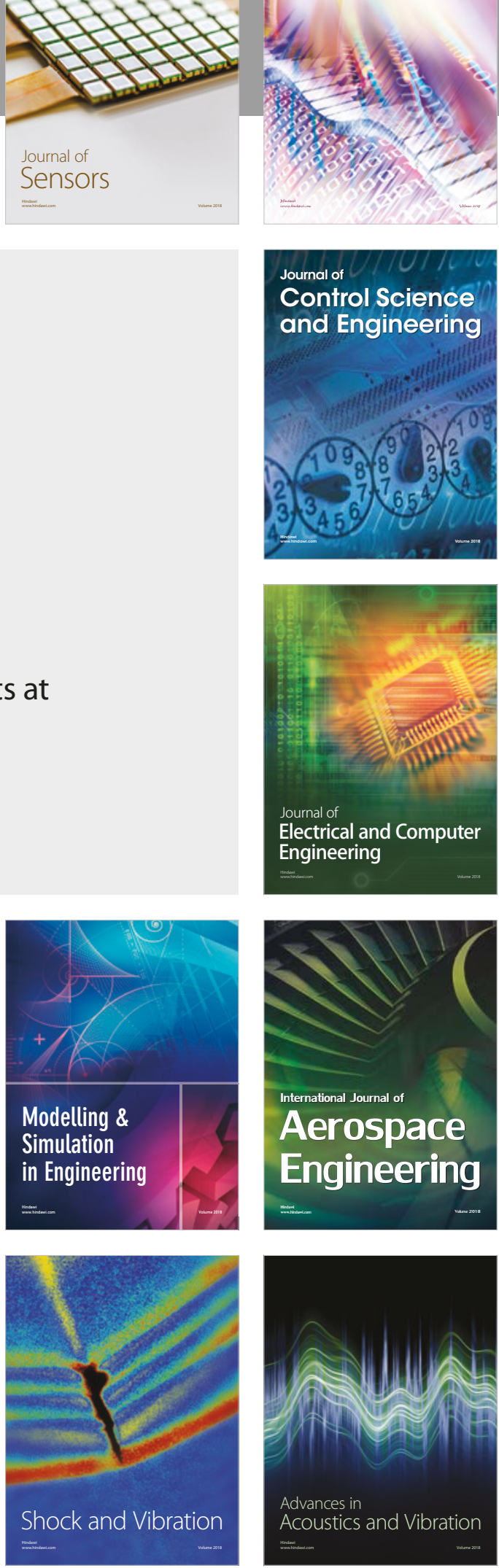